\title{
A importância da participação social para os Comitês de Ética em Pesquisa com
}

\section{seres humanos}

The importance of social participation for Research Ethics Committees involving Human

La importancia de la participación social para los Comités de Ética en investigaciones con humanos

Recebido: 28/08/2021 | Revisado: 04/09/2021 | Aceito: 09/09/2021 | Publicado: 12/09/2021

\author{
Alexandre Palma \\ ORCID: https://orcid.org/0000-0002-4679-9191 \\ Universidade Federal do Rio de Janeiro, Brasil \\ E-mail: palma_alexandre@yahoo.com.br \\ Carlos Alberto Guimarães \\ ORCID: https://orcid.org/0000-0002-7657-628X \\ Universidade Federal do Rio de Janeiro, Brasil \\ E-mail: caguimaraes@gbl.com.br
}

\begin{abstract}
Resumo
O objetivo do presente ensaio é apontar para a importância da participação da sociedade no "controle social" das atividades científicas, envolvendo seres humanos no Brasil. A legislação que trata e regula a ética em pesquisa com seres humanos no país, de forma bastante relevante e progressista, destaca a necessidade da participação social no controle do Sistema e inclui o representante de participantes de pesquisa. Historicamente, desde as primeiras orientações éticas, as quais coincidiram com a abertura democrática no país, houve o entendimento da necessidade da participação social no sistema que regula a ética em pesquisa com seres humanos. Tal compreensão resultou, recentemente, na Resolução n. 647, que “dispõe sobre as regras referentes à regulamentação do processo de designação e atuação dos membros de CEP indicados por entidades do controle social". Nesse sentido, a participação dos representantes de participantes de pesquisa pode ser considerada um importante avanço de nossa legislação que trata das pesquisas científicas e significar uma relevante posição na tentativa de assegurar o respeito à vida daqueles que se submetem às pesquisas científicas com o intuito de colaborar com o avanço da ciência.
\end{abstract}

Palavras-chave: Controle social; Comitês de ética em pesquisa; Bioética.

\begin{abstract}
The aim of this essay is to point out the importance of society's participation in the "social control" of scientific activities involving human beings in Brazil. The Brazilian legislation that addresses and regulates ethics in research with human beings, in a very relevant and progressive way, highlights the need for social participation in the control of the System and includes the representative of research participants. Historically, since the first ethical guidelines, which coincided with the democratic opening in the country, there was an understanding of the need for social participation in the system that regulates ethics in research with human beings. This understanding resulted, recently, in Resolution n. 647, which "disposes about the rules regarding the regulation of the process of designation and performance of CEP members appointed by social control entities". Thereby, the participation of representatives of research participants can be considered an important advance in our legislation that addresses scientific research and to signify a relevant position in an attempt to ensure respect for the lives of those who participate in scientific research in order to collaborate with the advancement of science.
\end{abstract}

Keywords: Social control; Research Ethics Committees; Bioethics.

\section{Resumen}

El objetivo de este ensayo es señalar la importancia de la participación de la sociedad en el "control social" de las actividades científicas que involucran al ser humano en Brasil. La legislación que trata y regula la ética en la investigación con seres humanos en el país, de manera muy relevante y progresiva, destaca la necesidad de la participación social en el control del Sistema e incluye al representante de los participantes de la investigación. Históricamente, desde los primeros lineamientos éticos, que coincidieron con la apertura democrática en el país, se comprendió la necesidad de participación social en el sistema que regula la ética en la investigación con seres humanos. Este entendimiento resultó, recientemente, en la Resolución n. 647, que "prevé las normas relativas a la regulación del proceso de designación y desempeño de los miembros del CEP designados por las entidades de control social". En este sentido, la participación de representantes de las investigaciones participantes puede ser considerada un avance importante en nuestra legislación que se ocupa de la investigación científica y significa una posición relevante en el intento de asegurar el respeto a la vida de quienes se someten a la investigación científica para poder colaborar, con el avance de la ciencia.

Palabras clave: Control social; Comités de Ética em investigación; Bioética. 


\section{Introdução}

O desenvolvimento de pesquisas com bases biomédicas se amparou firmemente em experiências, à luz dos pressupostos bioéticos da atualidade, bastante questionáveis. Nesse sentido, são vários os exemplos de investigações em que os participantes foram tratados como "cobaias", tal como as cobaias "não humanas". Os casos envolveram especialmente grupos sociais vulneráveis e de classe social considerada não importante para a sociedade, tais como os prisioneiros, os negros ou pessoas em condições de pobreza (Rego, Palácios \& Siqueira-Batista, 2009).

Dois grandes exemplos, muito debatidos nos estudos sobre bioética, dizem respeito à pesquisa sobre sífilis, em Tuskegee, que recrutou negros e negras, deixou-os sem tratamento, quando já havia a penicilina e não os informou claramente para que consentissem na realização da pesquisa de forma livre e esclarecida; bem como, os experimentos nazistas. Exemplos de más condutas em investigações científicas, igualmente, ocorreram no Brasil, como as pesquisas envolvendo esquistossomose mansônica, em que os participantes receberam placebo e ficaram sem o devido tratamento (Rego et al., 2009).

Esses casos ilustram uma preocupação já assinalada no código de Nuremberg e, posteriormente, na Declaração de Helsinque, qual seja a necessidade de regulamentar as pesquisas em humanos e, especialmente, de proteger os participantes a elas submetidos. Tal posição está em conformidade com os princípios bioéticos da não maleficência e da autonomia. O primeiro implica no dever de abster-se de fazer qualquer mal aos participantes, de não causar danos ou colocá-los em risco (Primum non nocere - primeiro não prejudicar). Desse modo, o pesquisador deveria comprometer-se a avaliar e evitar os danos previsíveis. O segundo diz respeito à autodeterminação ou autogoverno, ao poder de decidir sobre si mesmo e preconiza que a liberdade de cada ser humano deva ser resguardada, além dos indivíduos poderem estar capacitados para deliberar sobre suas escolhas (Campos \& Oliveira, 2017).

É a partir desses pressupostos, portanto, que a legislação brasileira que trata das pesquisas científicas, envolvendo seres humanos, estabelece a presença dos representantes dos participantes de pesquisa no colegiado dos Comitês de Ética em Pesquisa.

Isso posto, objetiva-se, neste curto ensaio, apontar para a importância da participação da sociedade no "controle social" das atividades científicas, envolvendo seres humanos no Brasil.

\section{Método}

De acordo com Marconi \& Lakatos (2017), o presente trabalho pode ser caracterizado como um ensaio crítico. Nesse tipo de trabalho procura-se realizar a exposição crítica de determinado tema, problematizando-o a partir de uma base teórica fundada na literatura científica vigente. Desse modo, o ensaio se caracteriza como um exercício de reflexão, mas os autores, ainda que tenham certa liberdade de posicionamento, não podem dispensar o rigor lógico e a coerência argumentativa.

\section{A Legislação Bioética no Brasil e o Controle Social}

A participação social em saúde somente se tornou realidade após o texto Constitucional incorporar o direito à saúde. Isso, porém, não se deu ao acaso e foi resultado da participação da sociedade civil em Conferências e Conselhos de Saúde. Portanto, somente a partir desse momento, em que os Conselhos de Saúde foram incluídos na estrutura decisória e no processo fiscalizatório dos recursos destinados ao Sistema Único de Saúde, é que o controle social tomou forma, de fato, e representaram importantes avanços no exercício do controle social, ainda que haja dificuldades reais para operacionalizá-lo (Shimizu \& Moura, 2015).

A noção de "controle social" pode ser compreendida como a participação e controle da sociedade civil para monitorar, fiscalizar, analisar e interferir na gestão estatal (Lima, Lima, Malacarne \& Cristofoletti, 2021). Segundo Fox (2019), as 
estratégias estabelecidas de controle social buscam aperfeiçoar a atuação institucional, fortalecendo a participação cívica e a capacidade do Estado em responder aos anseios dos cidadãos. Portanto, ao dar voz e força ao cidadão frente ao Estado, o controle e a participação social fariam parte de um processo político. Por outro lado, diferente da situação em que a voz dos cidadãos é delegada a outrem, como no caso dos representantes eleitos, o controle social assume especial relevância para a sociedade.

A participação social nas análises éticas em pesquisa envolvendo seres humanos tem razão de ser. Toda e qualquer pesquisa envolve risco, ainda que de menor potencial ofensivo. A noção de risco é de fundamental importância, na medida em que poderia impactar a integridade física, moral, psíquica, intelectual, social ou espiritual dos participantes. Nesse sentido, o objetivo primeiro de todo sistema de regulação da ética em pesquisa pressupõe a proteção dos participantes de pesquisa e a necessidade primordial de evitar que danos possam ser causados a eles (Lemos \& Aquino, 2021).

Para Rego et al. (2009), a emergência de uma legislação bioética no Brasil só veio a acontecer depois da promulgação da Constituição Federal de 1988, não coincidentemente em um ambiente de maior liberdade e respeito aos direitos individuais e coletivos. Desta forma, um dos primeiros marcos foi a aprovação, pelo Conselho Nacional de Saúde (CNS), da Resolução CNS n. 1 de 1988, como uma primeira tentativa de regulamentar a ética em pesquisa envolvendo seres humanos no Brasil.

Alguns pontos são importantes ao tratar dessa Resolução, ainda que indiretamente. Primeiro, é preciso destacar que na VIII Conferência Nacional de Saúde, realizada em 1986, em seu tema sobre a reformulação do Sistema Nacional de Saúde, já se anunciara, nos itens 24 e 25, a constituição de um novo Conselho Nacional de Saúde (CNS) com a possibilidade de alguma participação social (Brasil, 1986, pp. 17-18):

24- Será constituído um novo Conselho Nacional de Saúde, composto por representantes dos Ministérios da área social, dos governos estaduais e municipais e das entidades vivis de caráter nacional, como partidos políticos, centrais sindicais e movimentos populares, cujo papel principal será o de orientar o desenvolvimento e avaliar o desempenho do Sistema Único de Saúde, definindo políticas, orçamento e ações.

25- Deverão também ser formados Conselhos de Saúde em níveis local, municipal, regional e estadual, compostos de representantes eleitos pela comunidade (usuários e prestadores de serviço), que permitam a participação plena da sociedade no planejamento, execução e fiscalização dos programas de saúde. Deverá ser garantida a eleição das direções das unidades do sistema de saúde pelos trabalhadores desses locais e pela comunidade atendida.

Um segundo aspecto se refere ao fato de que, a despeito da abertura para a maior participação social, a Resolução 01, do Conselho Nacional de Saúde, de 1988 (Brasil, 1988) não avança quanto à participação de representantes dos usuários do sistema de saúde. Por fim, embora a referida Resolução seja um importante marco legal, não apresentou um impacto significativo na comunidade científica, possivelmente em decorrência do modo pouco participativo com que foi elaborada (Rego et al., 2009).

A Resolução 196, de 10 de outubro de 1996, por outro lado, aprovada após inúmeros debates com diferentes atores sociais, avançou ao trazer para a composição dos Comitês de Ética em Pesquisa (CEPs) o representante do usuário do sistema de saúde. O item VII.4., que trata da Composição do CEP, afirma:

O CEP deverá ser constituído por colegiado com número não inferior a 7 (sete) membros. Sua constituição deverá incluir a participação de profissionais da área de saúde, das ciências exatas, sociais e humanas, incluindo, por exemplo, juristas, teólogos, sociólogos, filósofos, bioeticistas e, pelo menos, um membro da sociedade representando os usuários da instituição. Poderá variar na sua composição, dependendo das especificidades da instituição e das linhas de pesquisa a serem analisadas (Brasil, 1996).

A participação de um membro representante dos usuários estava, igualmente, garantida no colegiado da Comissão Nacional de Ética em Pesquisa (Conep). 
Outro importante passo da Resolução 196 foi a proposta de regulação ética por meio dos CEPs com seres humanos, estabelecidos em diferentes instituições, sob a coordenação da Conep. Ademais, a Conep estaria estrategicamente vinculada ao CNS, uma vez que se acreditava proteger o Sistema CEP/ Conep de todos e quaisquer tipos de pressão e potenciais interesses, seja de ordem política, corporativa ou mercadológica. Estar articulada ao CNS conferiu à Conep maior êxito em efetivar o controle social das atividades de pesquisa e independência em relação ao Ministério da Saúde, ambos, controle social e independência, garantidos pela Carta Magna de 1988 e reafirmados nas Conferências Nacionais de Saúde (Harayama, 2011). Ademais, cabe destacar que o sistema de avaliação ética no Brasil funciona exatamente como um Sistema e não como um conjunto de comitês de ética que atuam isoladamente (Palácios \& Rego, 2015).

Portanto, como advoga Harayama (2011), a grande novidade é a constituição de um sistema de regulação bioética, que envolve diferentes comitês de ética e a coordenação de uma Comissão hierarquicamente superior (sistema CEP/ Conep), baseada no controle social. Tal situação implicou que pesquisadores de diferentes níveis e prestígio tivessem suas propostas de pesquisas analisadas por um comitê independente, formado por profissionais de diferentes disciplinas e, ao menos, um membro representante dos usuários do sistema de saúde.

O propósito desse arranjo de controle social da produção científica, portanto, é, em última instância, respeitar a dignidade humana e assumir como base a compreensão de que é preciso não provocar malefícios aos participantes de pesquisa (Harayama, 2011). Não por acaso, o Sistema de regulação ética brasileiro, por sua independência, controle social e participação democrática, tem recebido o merecido reconhecimento internacional (Palácios \& Rego, 2015).

Para Harayama (2011), se o aspecto central do funcionamento do Sistema CEP/ Conep diz respeito à proteção ao participante de pesquisa, é preciso que esse esteja representado nas discussões do Colegiado dos CEPs. O representante de participante de pesquisa se torna fundamental por estar desvinculado das instituições acadêmicas, dos próprios pesquisadores e dos patrocinadores. Assim, o representante poderia, com o principal objetivo de defender o participante de pesquisa, exercer o efetivo controle social das atividades científicas. Por outro lado, talvez esse seja o grande desafio a ser transposto, uma vez que não é rara sua subutilização ou mesmo sua substituição por profissionais do meio acadêmico.

A regulamentação bioética, como não poderia deixar de ser, está em constante aperfeiçoamento. Desse modo, mais recentemente a Resolução 196/96 foi revogada e deu lugar à resolução 466, de 12 de dezembro de 2012 (Brasil, 2012). Cabe destacar que essa mudança ocorreu a partir de uma consulta pública com diferentes atores sociais. Em norma operacional que a acompanha (Norma Operacional 001, de 2013) é reafirmada a participação do representante de participante de pesquisa:

Da composição: o CEP será composto por, no mínimo, sete (7) membros, dentre eles, pelo menos, um representante de usuários, respeitando-se a proporcionalidade pelo número de membros. Pelo menos 50\% dos membros deverão comprovar ter experiência em pesquisa. Poderá variar na sua composição, de acordo com as especificidades da instituição e dos temas de pesquisa a serem analisados. Terá, sempre, caráter multidisciplinar, não devendo haver mais que a metade dos seus membros pertencente à mesma categoria profissional, participando pessoas dos dois sexos. Poderá, ainda, contar com consultores "ad hoc", pertencentes, ou não, à instituição, com a finalidade de fornecer subsídios técnicos.

1. Da indicação do membro usuário: a indicação da representação de usuários é feita, preferencialmente, pelos Conselhos Municipais ou Estaduais de Saúde, cabendo ao CNS, por meio da CONEP, contribuir no processo de fortalecimento da participação dos representantes de usuários. A indicação do usuário também poderá ser feita por movimentos sociais, entidades representativas de usuários e encaminhadas para a análise e aprovação da CONEP.

De modo semelhante, a Resolução 510, de 07 de abril de 2016 (Brasil, 2016), que dispõe sobre as normas aplicáveis a pesquisas com metodologias das Ciências Humanas e Sociais (CHS), foi resultado de amplo debate, especialmente, com os profissionais desse campo de conhecimento. De fato, os representantes das CHS se mobilizaram e buscaram normativa éticas específicas para o desenvolvimento de investigações científicas nesse campo. Assim, consultas públicas e debates foram extensivamente realizados, antes da promulgação da Resolução 510/16. 
Um aspecto interessante, trazido à tona na $5^{\text {a }}$ edição da Conferência Nacional de Saúde Indígena, ocorrida em 2014 diz respeito ao reconhecimento da importância e necessidade de fiscalizar-se e acompanhar, com maior cuidado, as pesquisas envolvendo indígenas. Ademais, está destacado, no referido relatório, a intenção dos povos indígenas em participar mais efetivamente das etapas das atividades científicas e assumir certo controle social (Gusman, Rodrigues \& Villela, 2016).

\section{Sobre os Membros Representantes dos Participantes de Pesquisa}

Os representantes dos participantes de pesquisa, outrora denominados de "representantes dos usuários", para composição dos CEPs têm sido essenciais para que se dê voz àqueles que se utilizam do sistema de saúde e podem, em algum momento, tomar parte de alguma pesquisa científica.

A escolha do representante pode ser solicitada junto ao Conselho Municipal de Saúde, associações de usuários já previamente estabelecidas ou outras associações da sociedade civil.

De acordo com o Manual Operacional para Comitês de Ética em Pesquisa (Brasil, 2002), o representante não deve exercer cargo na instituição e não ser profissional de saúde. Além disso, deve ser alguém empenhado no estudo da ética em pesquisa com seres humanos e na defesa dos direitos das pessoas e usuários do sistema de saúde, mostrando capacidade de contribuir nos debates dos protocolos específicos, representando os interesses e preocupações da sociedade. Sobre essa questão Lima et al. (2021) destacam que o representante do participante de pesquisa é um importante membro no controle social do Sistema, muito embora os autores mencionem que a exigência estava explícita, mas não definida, uma vez que não estavam claras as competências desse membro.

Contudo, em 12 de outubro de 2020, o Conselho Nacional de Saúde publicou a Resolução n. 647, que "dispõe sobre as regras referentes à regulamentação do processo de designação e atuação dos membros de CEP indicados por entidades do controle social". A referida Resolução aprova, entre outras regras, que o representante de participantes de pesquisa "deve possuir histórico de participação em movimento social elou comunitário", que, decerto modo, visa garantir a experiência na luta política e participação social; "fomentar, em colaboração com os demais membros do Sistema CEP/Conep, questões específicas relacionadas aos interesses e direitos dos participantes de pesquisa" e "contribuir na avaliação ética desenvolvida pelo CEP, podendo realizar a relatoria de protocolos de pesquisa, quando assim for designado pela coordenação do CEP", buscando assegurar o poder político nas reuniões dos CEPs em relação aos outros membros; além de determinar que o CEP deva "prever Plano de Capacitação Permanente para os seus membros, devendo incluir conteúdo direcionado e acessível aos representante de participantes de pesquisa" e "estimular o protagonismo dos RPP respeitando suas características individuais" (Brasil, 2020).

Ademais, recentemente, a Conep publicou uma Cartilha dos Direitos dos Participantes de Pesquisa, explicando como ocorrem as pesquisas com seres humanos no país, quais são os órgãos competentes na regulação, normalização e educação, bem como, na tentativa de proporcionar segurança e autonomia aos participantes de pesquisa. O referido documento, desenvolvido em linguagem simples, auxilia na compreensão dos direitos e deveres de cada um envolvido com a pesquisa.

Entre os direitos relacionados à proteção dos participantes de pesquisa encontram-se a necessidade de recebimento de informações sobre a/o: i) pesquisa, de modo claro e acessível; ii) assistência gratuita, integral e imediata por danos, quando for necessária; iii) confidencialidade dos dados; iv) ressarcimento de todas e quaisquer despesas decorrente da participação na pesquisa; entre outros.

Cabe destacar que a legislação que trata da representação dos participantes de pesquisa em outros países tem apresentado diferenças importantes. Na França, por exemplo, diferentemente da maior parte dos países membros da União Europeia, não há exigência legal para a representação do participante de pesquisa (Lima et al., 2021). Lima et al. (2021) ressaltam que frequentemente o estabelecimento dos representantes ocorre em função de "negações". Assim, na Suécia, 
Dinamarca e Reino Unido, o representante do participante de pesquisa não pode ser profissional de saúde; Na Bélgica, é vedada a participação de trabalhadores de hospitais; Na Noruega, estão proibidos os profissionais de saúde que representam hospitais, bem como advogados e psicólogos envolvidos com grupos específicos de pacientes, uma vez que poderiam representar interesses setoriais particulares. No Brasil, a recente Resolução 647/2020, em seu artigo quarto, que primeiro trata do perfil e atuação do representante de participantes de pesquisa (Brasil, 2020), determina que o representante "deve possuir histórico de participação em movimento social elou comunitário".

Lima et al. (2021), em concordância com Foucault, apontam para o risco de a participação social poder comprometerse e tornar-se cúmplice dos poderes vigentes, ao envolver-se dentro do próprio processo. Assim, os representantes podem, quando não gozarem de plena autonomia e liberdade, ser levados a interpretar as normas de acordo com as forças indesejáveis e tornarem-se cúmplices de processos eticamente inadequados. Ademais, talvez seja preciso começarmos a pensar, como salientaram Escorel \& Arouca (2016), o processo democrático e a participação social para além daquilo previamente estabelecido, necessitando de mecanismos complementares entre os canais institucionais e modos mais fluidos de participação social nos assuntos de interesse de toda sociedade.

Por fim, cabe destacar que a ética não se estabelece por decreto e não deveria estar presa às medidas burocráticas que, por vezes, fazem parte da legislação (Lima \& Lima, 2021). Além disso, uma "ética prescritiva", constituída a partir de uma espécie de "contrato" assegurado pela legislação vigente, ainda que necessária, pode não ser suficiente para garantir a adequada postura ética dos pesquisadores (Pereira, Guareschi \& Machado, 2019) e mesmo dos membros dos comitês de ética em pesquisa. Por essa razão, torna-se fundamental que o envolvimento do representante do participante de pesquisa não ocorra como um mero instrumento burocrático para cumprir a legislação.

\section{Considerações Finais}

A participação dos representantes de participantes de pesquisa é considerada um importante avanço de nossa legislação que trata das pesquisas científicas envolvendo seres humanos. Tal participação pode significar uma relevante posição na tentativa de assegurar o respeito à vida daqueles que se submetem às pesquisas científicas com o intuito de colaborar com o avanço da ciência.

Por outro lado, é preciso reconhecer que, em muitos CEPs, os representantes têm sido subutilizados ou mesmo alijados do processo, o que pode tornar fragilizada a situação do Sistema. Nesse sentido, é preciso que estudos futuros procurem abordar o trabalho cotidiano dos representantes de participantes de pesquisa, bem como, o reflexo da Resolução 647/2020 sobre o sistema CEP/ Conep.

\section{Referências}

Brasil. (1988). Resolução n. 01 do Conselho Nacional de Saúde. Brasília, 13 jun. 1988.

Brasil. (1996). Resolução 196, de 10 de outubro de 1996. Brasília, 10 out. 1996. https://bvsms.saude.gov.br/bvs/saudelegis/cns/1996/res0196_10_10_ 1996.html.

Brasil. (2002). Manual Operacional para Comitês de Ética em Pesquisa. da Comissão Nacional de Ética em Pesquisa. Brasília: Ministério da Saúde, 2002. https://conselho.saude.gov.br/biblioteca/livros/Manual_ceps.pdf.

Brasil. (2012). Resolução 466, de 12 de dezembro de 2012. Brasília, 12 dez. 2012. https://bvsms.saude.gov.br/bvs/saudelegis/cns/ 2013/res0466_12_12_2012.html.

Brasil. (2016). Resolução 510, de 07 de abril de 2016. Brasília, 07 abr. 2016. https://bvsms.saude.gov.br/bvs/saudelegis/cns/2016/res0510_07_04_2016.html.

Brasil. (2020). Resolução 647, de 12 de outubro de 2020. Brasília, 12 out. 2020. http://conselho.saude.gov.br/resolucoes-cns/resolucoes-2020/1422-resolucaon-647-de-12-de-outubro-de-2020.

Brasil. (1986). VIII Conferência Nacional de Saúde. Relatório final. 1986. <https://bvsms.saude.gov.br/bvs/publicacoes/8_conferencia_nacional_s aude_relatorio_final.pdf. 
Research, Society and Development, v. 10, n. 12, e10101219973, 2021

(CC BY 4.0) | ISSN 2525-3409 | DOI: http://dx.doi.org/10.33448/rsd-v10i12.19973

Campos, A. \& Oliveira, D. R. (2017). A relação entre o princípio da autonomia e o princípio da beneficência (e não-maleficência) na bioética médica. Revista Brasileira de Estudos Políticos, 115, 13-45.

Escorel, S. \& Arouca, L. E. (2016). Democracia e participação: para além das dicotomias. Saúde Debate, 40 (n. especial), 39-48.

Fox, J. (2019). Controle Social: o que as evidências realmente mostram?. Revista da CGU, 11 (20), 1331-1359.

Gusman, C. R., Rodrigues, D. A. \& Villela, W. V. (2016). Trâmites éticos, ética e burocracia em uma experiência de pesquisa com população indígena. Saúde Soc, 25 (4), 930-942.

Harayama, R. M. (2011). Do ponto de vista do sujeito da pesquisa: Evento e Cultura material em um Comitê de Ética em Pesquisa (Master's dissertation, Universidade Federal de Minas Gerais), Belo Horizonte, Brasil.

Lemos, P. B. S. \& Aquino, F. J. A. (2021). O conceito de risco nas resoluções brasileiras que regulamentam a revisão ética da pesquisa envolvendo seres humanos. Research, Society and Development, 10 (7), e23110715917.

Lima, D. F. \& Lima, L. A. (2021). Perspectivas da ética em pesquisa: o repensar para o futuro do sistema normatizador brasileiro. Cadernos UniFOA, 45, 8995 .

Lima, D. F., Lima, L. A., Malacarne, V. \& Cristofoletti, J. F. (2021). O lugar do representante do controle social nos comitês de ética em pesquisa brasileiros. Revista de Bioética y Derecho, 52, 253-264.

Marconi, M. A. \& Lakatos, E. M. (2017). Fundamentos de Metodologia Científica. Atlas.

Palácios, M. \& Rego, S. (2015). A proposta de regulamentação ética da pesquisa clínica apresentada ao Senado Brasileiro não interessa aos participantes de pesquisa. Cad. Saúde Pública, 31 (8), 1583-1585.

Pereira, C. C. Q., Guareschi, P. A. \& Machado, F. V. (2019). Ética e participação na prática de pesquisa: contribuições para o pensamento crítico em Psicologia Social. In D. Perez (Coord.). Coleção Encontros em Psicologia Social. Psicologia Social Crítica: tecendo redes e articulando resistências em contextos de retrocessos (Vol. VI, Cap. 5, p. 83-97). Porto Alegre: Abrapso.

Rego, S., Palácios, M. \& Siqueira-Batista, R. (2009). Bioética: histórico e conceitos. Bioética para profissionais da saúde. Rio de Janeiro: Fiocruz.

Shimizu, H. E. \& Moura, L. M. (2015). As representaç̃̃es sociais do controle social em saúde: os avanços e entraves da participação social institucionalizada. Saúde Soc, 24 (4), 1180-1192. 